\title{
Are Artificial Intelligence (AI) And Machine Learning (ML) Having An Effective Role In Helping Humanity Address The New Coronavirus Pandemic?
}

\author{
*KARIMAN RAMZY El HELOW and **ABDEL-BADEEH M. SALEM \\ *Higher Institute of Computer, King Mariout, Alexandria, King Mariout Academy, Alexandria, \\ EGYPT \\ **Faculty of Computer and Information sciences, Ain Shams University, Cairo, EGYPT
}

Abstract. COVID-19 has become a pandemic affecting the most of countries in the world. One of the most difficult decisions doctors face during the Covid-19 epidemic is determining which patients will stay in hospital, and which are safe to recover at home. In the face of overcrowded hospital capacity and an entirely new disease with little data-based evidence for diagnosis and treatment, the old rules for determining which patients should be admitted have proven ineffective. But machine learning can help make the right decision early, save lives and lower healthcare costs. So, there is therefore an urgent and imperative need to collect data describing clinical presentations, risks, epidemiology and outcomes.

On the other side, artificial intelligence(AI) and machine learning(ML) are considered a strong firewall against outbreaks of diseases and epidemics due to its ability to quickly detect, examine and diagnose these diseases and epidemics. AI is being used as a tool to support the fight against the epidemic that swept the entire world since the beginning of 2020 ..

This paper presents the potential for using data engineering, ML and AI to confront the Coronavirus, predict the evolution of disease outbreaks, and conduct research in order to develop a vaccine or effective treatment that protects humanity from these deadly diseases.

\section{KEYWORDS. COVID-19, SARS-CoV-2, Variation, Artificial intelligence, Machine Learning}

Received: March 26, 2020. Revised: September 30, 2020. Accepted: October 28, 2020. Published: November 18, 2020.

\section{INTRODUCTION}

COVID-19 emerged in Wuhan, China, in December 2019. COVID-19 is the infectious disease caused by the most recently discovered coronavirus. New coronavirus (COVED-19) is a new strain of Coronavirus that no human being knew before. Coronaviruses are a large family of viruses known to cause diseases ranging from the common cold to more serious illnesses such as Severe Acute Respiratory Syndrome (SARS).Some reports indicated that more than $50 \%$ of emerging infectious diseases are of animal origin. They are transmitted from animals to humans, and it has been proven that the SARS virus was transmitted from civet cats to humans in China in 2002, or in bats in the event of infection with the current Coronavirus.

COVID-19, also known as SARS-CoV-2, is a new virus that infects humans. It appeared in Wuhan, China, in December 2019. Covid-19 has become a pandemic that affects every country in the world[1].Investigations found that the many known corona viruses spread in animals or bats, as is the case in infection with the new Corona virus [2].
COVID-19 spreads from person to person through small drops from the nose or mouth, which is expelled when a person with COVID-19 coughs, sneezes or speaks [3]. These drops are relatively heavy, and they do not travel far distances in the air but it do land quickly on the ground. This is why it is important for people to stay at least one meter away from each other. Also, these drops may land on surfaces and objects around persons, such as doorknobs, handrails and tables. Accordingly, it is possible for people may become infected by touching these surfaces or objects, and then touching their mouth, nose, eyes, or face. For this reason, it was important to wash hands thoroughly and regularly with soap and water, or clean them with an alcohol disinfectant. But common signs of this disease include respiratory symptoms, fever, cough, shortness of breath and difficulty breathing, and infection can cause severe pneumonia, kidney failure and even death. So it is necessary to take into account the precautionary precautions and wearing the masks. The time between exposure to COVID- 
19 and the onset of symptoms can take Period of time from five to six days but can range from one to 14 days [4 - 7]. There is a significant relationship between spread control and the degree of population control. This is due to two main reasons: the degree of cultural awareness of the people, and the abdication of privacy and personal freedom, for the sake of public health, for example that countries such as Singapore, China and South Korea have all adopted policies of strict closure, surveillance, social distancing and the protection of isolation in case of suspected epidemic Which helped stop the virus from progressing. In contrast to countries that adopts a policy of privacy and personal freedom, which led to the spread of this epidemic in them [8].

Artificial intelligence (AI) is one of the ways or means to understand the virus, developing preventive and control measures[9], using mathematical modeling to understand virus transmission, structural biology to determine the structure of the virus and developing vaccines [10], computational biology to understand virus evolution, as well as cohesion studies to examine drugs and inhibitors[11].

The aim of this paper is to use artificial intelligence as a method or methods to understand the virus, develop preventive measures, develop the necessary vaccines, and test drugs to provide appropriate treatment. This paper discusses the potential role of artificial intelligence and machine learning in helping humanity cope with the novel coronavirus pandemic.

The rest of the paper is organized as follows: Section 2 COVID19 World Health Organization reports, Section 3 the Potential role of AI and ML in the betel against COVID-19, Section 4 provides results and discussion of a brief overview of Corona disease and known AI technologies, and discusses the implications of using AI in such diseases. The final section concludes the paper and explains future work.

\section{COVID19 World Health Organization Reports}

COVID-19 is an infectious disease caused by the most recently discovered corona virus. This new virus and disease were not known before the outbreak. No vaccine has been discovered yet. The development of a new vaccine may take a period of time that may reach several years. There is also no specific treatment for the disease caused by the new Corona virus. However, many symptoms can be treated and therefore treatment depends on the patient's clinical condition. According to the statistics of the World Health Organization, the number of people infected with this virus and the number of deaths, as shown below: $[12,13]$

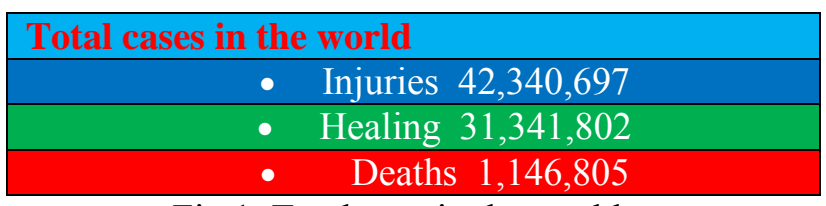

Fig 1, Total case in the world

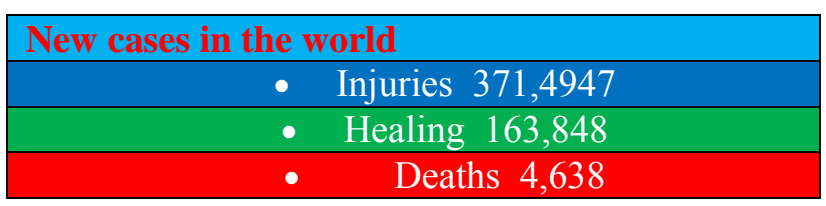

Fig 2, New cases in the world

\begin{tabular}{|l|l|}
\hline Active Cases & $9,852,090$ \\
\hline Critical Cases & 75,910 \\
\hline Closed Cases & $32,488,607$ \\
\hline Recovery Rate & $74.0 \%$ \\
\hline Death Rate & $2.7 \%$ \\
\hline
\end{tabular}

Tabel 1 , the percentage of cases

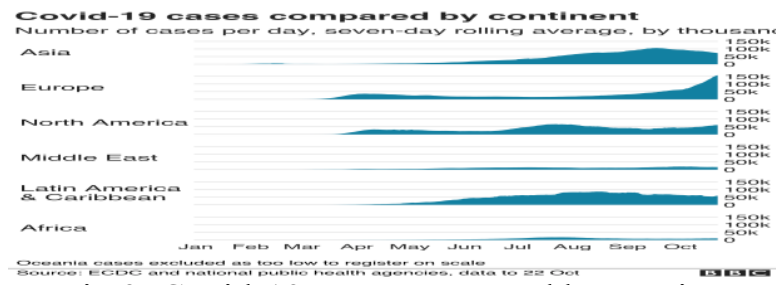

Fig 3, Covid-19 cases compared by continent, data to 22 Oct

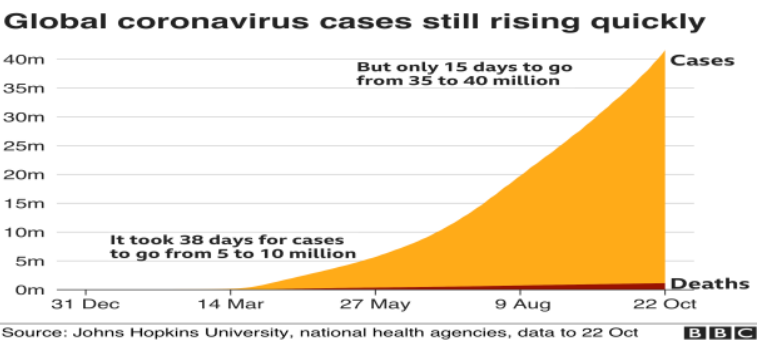

Fig 4, high incidence Coronavirus

Reports have shown that the elderly and those who suffering from health problems such as diabetes, cardiovascular disease, Pressure diseases, cancer and chronic respiratory diseases and kidney failure are more likely to develop serious diseases [14] .

According to most announced reports, more than $50 \%$ of infectious diseases emerging or re-emerging are of zoonotic origin; they are transmitted from animals to humans, directly or through intermediate hosts. Such as bird flu, SARS / MERS / SARS-2 coronavirus, and many other viruses. So, viruses are studied in their natural hosts, whether in birds as in 
cases of influenza viruses, or in bats as in the case of infection with the new Corona virus [15].

More than thirty countries and many international institutions and companies have signed up to support the COVID-19 Technology Access Pool (CTAP) in an initiative to make vaccines, tests, treatments, and other health technologies within everyone's reach to combat this pandemic. The World Health Organization has coordinated efforts to develop vaccines and drugs to prevent and treat the COVID-19 virus and will continue to provide updated information as soon as research results become available [16].

\section{The Potential Role of AI and ML in the Betel against COVID-19}

Artificial intelligence and machine learning can search large databases for drugs currently available in the market that can be used against the emerging corona virus or can help develop a new vaccine and treatment for this new virus in the shortest period of time [17].While some conventional and commercially available treatments may provide relief and relieve mild symptoms of COVID-19, there are no medicines that have been shown to prevent or treat the disease. The World Health Organization does not recommend antibiotic treatment, as prevention or treatment for COVID19. This is because antibiotics only work on bacterial infections [18].

Big data is the key to technology. Machine learning and artificial intelligence techniques have been used to access data from many different sources such as social media, digital media, airline ticket data, news sources, and public health reports, processing and synthesize this data every 15 minutes to create standardized reports, Taking into account that there will be an artificial intelligencedriven textual analysis to work on removing misinformation programs from social media. Artificial intelligence-based models can also be developed and trained to analyze very large amounts of data from various sources, and the main strength of artificial intelligence methods is the implementation of tasks that require experts to work continuously and around the clock at high speed. This makes analysis more efficient and scalable, and complements human intelligence to support timely decision making. As with Coronavirus, artificial intelligence is expected to play an important role in early detection of this disease outbreak in the near future to combat it or limit its spread and save lives from death [19-21].

Artificial intelligence has the power to help in all stages of healthcare, from monitoring syndromes to rapid diagnostic tests and faster drug development. AI may also help determine which patients should be prioritized for treatment [22]. Machine learning techniques can also be trained to rapidly identify factors that predict higher risk of death [23], in addition to population-level interventions and controls that have minimized harm [24]. Artificial intelligence methods that incorporate genetic, biological and environmental data can help discover a cure for the novel coronavirus.

Biomedicine and research are based on computer science and statistics that have already contributed for a long time. Thus, the application of artificial intelligence in the face of health crises is to help researchers find a vaccine capable of protecting caregivers and containing an epidemic, predicting the development of disease outbreaks and searching for a vaccine or effective treatment. [25]

Artificial intelligence (AI)-based models are now being used to identify at-risk populations or people who should be screened first, also taking into account various scenarios and diseases, and coming to quarantine to separate people who are not sick but may have been exposed to COVID-19. While these people have symptoms, As well as resorting to isolation in the case of people who show symptoms of Covid-19 disease and who may already be contagious, this is to prevent the disease from spreading. And follow procedures to keep a distance of at least one meter from others, with the necessity of washing hands regularly with soap and water or cleaning them with an alcohol disinfectant. Most studies have shown that the COVID-19 virus can live for up to 72 hours on plastic and stainless steel, as little as 24 hours on cardboard and less than 4 hours on copper [26 - 27].

AI-based systems are already being used to diagnose many health conditions including detecting signs of disease, diabetes and retinopathy using ophthalmic images, detecting lesions in skin images, and assessing risk of sudden cardiac death using cardiac MRI and ECG images Electrophoresis. It appears that AI has provided significant support in this sense, even if somewhat limited, because it provides scientists with additional information about how viruses spread, risk factors, non-drug treatments, the genetic characteristics of the virus, and vaccine development efforts. Thus, the prediction of the secondary structure of the Covid19 RNA sequence was calculated by Linearfold at 27 seconds instead of 55 minutes. This is because of so-called "continuous" bases and infinite combinations of studying protein folding. While the time required to develop a prototype of a vaccine that can be tested in humans is significantly reduced 
thanks to the support of bioinformatics, of which AI is an integral part [28-29].

In a study of SARS CoV 2 genomic sequences it was found to be available in the NCBI and GISAID databases, and a genetic analysis of the viral genome (29,903 nucleotides) revealed that the virus was more closely related $(89.1 \%$ nucleotide similarity) to a group of SARS-like coronaviruses (genus Betacoronavirus, subgenus Sarbecovirus) Which has been previously found in bats in China, and this demonstrates the ability of this virus to continue to leak from animals to humans to cause this dangerous disease. The study of the genomic variation of SARS-CoV 2 is very important to know the course of the disease, ways to prevent it, and how to treat it, as the sequence of this virus facilitates viral detection, functional analysis, design of an effective vaccine, and evaluation of drug effectiveness[ 30- 32].

And in another study of the sequence alignment of virus strains, and analysis of the similarity of the genomic sequence SARS-CoV-2 with the reference sequence (NC_045512). It turns out that there are very high similarities between all the sequences but over time there has been a decrease in similarity. Also results are equivalent to the similarity between the SARS-CoV-2 sequences. Wang et al obtained a similarity of $99.99 \% \quad(99.91 \% \quad-100 \%)$ at the nucleotide level, between viral strains, and although the general similarity between the human strains was high, the similarity decreased later, indicating the occurrence of mutations within the human being. It can also be recognized that SARS-CoV-2 is a virus with a relatively low mutation rate, and this indicates a potential treatment solution. The pairwise alignment algorithm has shown high efficiency providing a faster methodology, thus machine learning methods can be very useful [3335].

\section{Results and Discussion}

Artificial intelligence can be used to use the new infrared sensor system to identify individuals who may be infected with COVID-19. By monitoring the temperature of nearby people and determining if they have a fever, as a high temperature in people is a symptom of the Corona virus. Artificial intelligence and big data have also been used to track contact and manage priority populations [36].

According to the preliminary results of the World Health Organization, which prove that the results of the initial clinical trials that treatment of dexamethasone, which is a corticosteroid, reduces the number of deaths infected with the Coronavirus, as it can save the lives of seriously ill patients from
COVID-19? For patients who use ventilators, it was found that treatment reduces deaths by about a third, and for patients who only need oxygen, the death rate has been reduced by about a fifth [37].

Donald Trump, President of the United States of America, unveiled an initiative to produce a vaccine for COVID-19 at the end of the year. Trump also financed this initiative with millions of dollars, which helped the race between countries and global companies to reach an effective vaccine for this epidemic [38].

Artificial intelligence, machine learning, and technology are being used to combat epidemics and identify, track and predict outbreaks and better track and control the virus. And understand how COVID19 spreads, and when, the infection rate reaches its peak. And it also helps diagnose the virus, and handle health care claims to reduce the amount of face-to-face interaction between patients, hospital staff and Attending physicians, and the provision of advanced fabrics that provide protection and others of face masks made of its anti-disease and antibacterial fabric.

To date, no drugs have been proven effective to treat COVID-19. However, a number of drugs have been proposed as potential treatments, and they will be studied in clinical trials, such as the SOLIDARITY trial sponsored by the World Health Organization and participating countries

\section{Conclusion and Future Work}

Artificial intelligence, machine learning, and big data analytics have become a firewall against epidemics and disease outbreaks due to their ability to quickly detect, screen and diagnose epidemics. Also, the use of artificial intelligence and machine learning are important tools to help fight the Coronavirus epidemic, which is an affecting the entire world. Machine learning and knowledge acquisition methods are used to detect Coronavirus compared to medical kits or CT scans based on data collected from smartphones. Artificial intelligence can also be integrated with thermal cameras, which are installed in smart cities for early detection of disease outbreaks. Artificial intelligence can also help support better decisions to contain dangerous viruses such as COVID-19.

Artificial intelligence tools can be used to better understand the chemistry and biology of the Coronavirus, in preparation for developing a vaccine and an effective treatment for such a dangerous virus, also the "AI-derived knowledge graph" of medical information has identified the group of approved drugs that can prevent viral infection of cells. In addition, the Benevolent AI 
Program has proven that baricitinib, approved for the treatment of rheumatoid arthritis, can be beneficial in combating the COVID-19 virus, along with appropriate clinical tests. And the use of artificial intelligence algorithms to design new molecules can limit the ability of COVID-19 to reproduce in cells.

In the future, the use of drones to deliver medical supplies to patients in need may be one of the safest and fastest ways to get medical supplies. Robots can also be used to sterilize and provide food and supplies to infected patients, as robots are not susceptible to contracting the virus. Blue Ocean Robotics' UVD robots use ultraviolet light to independently kill bacteria and viruses. It is also possible to use artificial intelligence systems to build drugs that are effective to fight incurable diseases in the world and help support efforts to treat the Corona virus. As it can be used to predict the spread of the coronavirus, map its genetic evolution as it is transmitted from one person to one or several other people, accelerate diagnosis, and develop potential treatments,

Every country must be keen to have an automated data processing center, in which records of patients affected by such epidemics, the clinical condition of each patient, symptoms indicating infection, and protocols for combating such diseases are placed in it, and there must also be information technology systems. Robust, secure and subject to transparent review, capable of handling volumes of data to be collected, processed and analyzed.

The question remains, will these destructive epidemics continue to appear due to human actions and their challenges to humans nature???

\section{Reference}

[1] Hui, D. S. et al. The continuing 2019-nCoV epidemic threat of novel coronaviruses to global health - the latest 2019 novel coronavirus outbreak in Wuhan, China. Intl. J. Infect. Dis. 91, 264-266 (2020).

[2] Zhou, P. et al. A pneumonia outbreak associated with a new coronavirus of probable bat origin. Nature 579, 70-273 (2020).

[3] $\mathrm{Wu} \mathrm{F}$, Zhao S, Yu B, et al. A new coronavirus associated with human respiratory disease in China. Nature. 2020;579:265-269.

[4] Zhang N,Wang L, Deng X. Recent advances in the detection of respiratory virus infection in humans. J Med Virol. 2020;92(4):408-417.

[5] Shu, Y. \& McCauley, J. GISAID: Global Initiative on Sharing All Influenza Data-from vision to reality. Eurosurveillance 22, 30494 (2017).
[6] Chan JFW, Yuan S, Kok KH, et al. A familial cluster of pneumonia associated with the 2019 novel coronavirus indicating person-to-person transmission: a study of a family cluster. Lancet. 2020;395:514-523.

[7] World Health Organization, Q\&A: How is COVID-19 transmitted?, 14 July 2020.

[8] Thornhill, J. (2020) -Coronairus monitoring poses dangers for civil liberties", [online], Financial Times, 23 March, www.ft.com/content/d6c877cc-6cfa-11 ea-9bcabf503995cd6f.

[9] Ye, Y., Hou, S., Fan, Y., Qian, Y., Zhang, Y., Sun, S., ... and Laparo, K. (2020). _-Satellite: An AI- driven system and benchmark datasets for hierarchical community-level risk assessment to help combat COVID-19. arXiv preprint arXiv:2003.12232.

[10] Rao, A. S. S., and Vazquez, J. A. (2020). Identification of COVID-19 can be quicker through artificial intelligence framework using a mobile phone-based survey in the populations when cities/towns are under quarantine. Infection Control and Hospital Epidemiology, 1-18. DOI: https://doi.org/10.1017/ice.2020.61.

[11] Bai, X., Fang, C., Zhou, Y., Bai, S., Liu, Z., Chen, Q., ... and Song, D. (2020). Predicting COVID-19 malignant progression with AI techniques.medRxiv, doi: https://doi.org/10.1101/2020.03.20.20037325.

[12] World Health Organization, WHO Coronavirus Disease (COVID-19) Dashboard, 2020.

[13] World Health Organization, Coronavirus disease (COVID-19)Weekly Epidemiological Update , 2020.

[14] Gerard Thomas Flaherty,corresponding author, Paul Hession, CheeHwuiLiew, Bryan Chang Wei Lim, Tan Kok Leong, Victor Lim, and Lokman Hakim Sulaiman, COVID-19 in adult patients with pre-existing chronic cardiac, respiratory and metabolic disease: a critical literature review with clinical recommendations, Tropical Diseases, Travel Medicine and Vaccines Journal, Vol. 6,2020.

[15] Zhou, P., Yang, X. L., Wang, X. G., Hu, B., Zhang, L., Zhang, W., ... and Chen, H. D. (2020). A pneumonia outbreak associated with a new coronavirus of probable bat origin. Nature, 579(7798), 270-273.

[16] World Health Organization, International community rallies to support open research and science to fight COVID-19, 2020.

[17] Li, L., Qin, L., Xu, Z., Yin, Y., Wang, X., Kong, B., ... and Cao, K. (2020). Artificial intelligence distinguishes COVID-19 from 
community acquired pneumonia on chest CT. Radiology, 200905.

[18] World Health Organization, Coronavirus disease (COVID-19) advice for the public: Mythbusters, 2020.

[19] Li, L., Zhang, Q., Wang, X., Zhang, J., Wang, T., Gao, T. L., ... and Wang, F. Y. (2020). Characterizing the propagation of situational information in social media during COVID-19 epidemic: A case study on Weibo.IEEE Transactions on Computational Social Systems.doi: 10.1109/TCSS.2020.2980007.

[20] Polonsky, J. A. et al. Outbreak analytics: a developing data science for informing the response to emerging pathogens. Philos. Trans. R. Soc. B 374, 20180276 (2019).

[21] European Union (2020) -EE SPECIAL REPORT UPDATE: SHORT ASSESSMENT OF NARRATIVES AND DISINFORMATION AROUND THE COVID-19 PANDEMIC (UPDATE 23 APRIL - 18 MAY)", [online], euvsdisinfo, 20 May, euvsdisinfo.eu/eeasspecialreport-update-short-assessment-of-narrativesand-disinformation-around-the-covid19pandemic-updated-23- april-18-may/.

[22]Becky McCall, COVID-19 and artificial intelligence: protecting health-care workers and curbing the spread,www.thelancet.com/digitalhealth Published online February 20, 2020 https://doi.org/10.1016/S2589-7500(20)30054-6

[23] Chowdhury, M. E., Rahman, T., Khandakar, A., Mazhar, R., Kadir, M. A., Mahbub, Z. B., ... and Reaz, M. B. I. (2020). Can AI help in screening viral and COVID-19 pneumonia?.arXiv preprint arXiv:2003.13145.

[24] Yan, L., Zhang, H. T., Xiao, Y., Wang, M., Sun, C., Liang, J., ...and Tang, X. (2020). Prediction of criticality in patients with severe Covid-19 infection using three clinical features: a machine learningbased prognostic model with clinical data in Wuhan. medRxiv, doi: https://doi.org/10.1101/2020.02.27.20028027.

[25] Jin, S., Wang, B., Xu, H., Luo, C., Wei, L., Zhao, W., ... and Sun, W. (2020). AI-assisted CT imaging analysis for COVID-19 screening: Building and deploying a medical AI system in four weeks. medRxiv, doi: https://doi.org/10.1101/2020.03.19.20039354.

[26] Hu, Z., Ge, Q., Jin, L., and Xiong, M. (2020). Artificial intelligence forecasting of Covid-19 in China.arXiv preprint arXiv:2002.07112.

[27] Feng, C., Huang, Z., Wang, L., Chen, X., Zhai, Y., Zhu, F., ... and Tian, L. (2020). A novel triage tool of artificial intelligence assisted diagnosis aid system for suspected COVID-19 pneumonia in fever clinics. Available at SSRN: http://dx.doi.org/10.2139/ssrn.3551355.

[28] Deng, S. Q. \&Peng, H. J. Characteristics of and public health responses to the coronavirus disease 2019 outbreak in China. J. Clin. Med. 9, 575 (2020).

[29] Chung, M., Bernheim, A., Mei, X., Zhang, N., Huang, M., Zeng, X., ... and Jacobi, A. (2020). CT imaging features of 2019 novel coronavirus (2019-nCoV). Radiology, 200230.

[30] Oude Munnink, B. B. et al. Towards high quality real-time whole genome sequencing during outbreaks using Usutu virus as example. Infect. Genet.Evol. 73, 49-54 (2019).

[31] Bas B. Oude Munnink, David F. Nieuwenhuijse, Rapid SARS-CoV-2 wholegenome sequencing and analysis for informed public health decision-making in the Netherlands, Nature Medicine Journal, 20 October, 2020.

[32] Li, H. Minimap2: pairwise alignment for nucleotide sequences. Bioinformatics 34, 30943100 (2018).

[33] Baichoo, S. and C. A. Ouzounis (2017). Computational complexity of algorithms for sequence comparison, short-read assembly and genome alignment.Biosystems 156-157: 72-85.

[34] Randhawa, G. S., M. P. M. Soltysiak, H. El Roz, C. P. E. de Souza, K. A. Hill and L. Kari (2020). Machine learning using intrinsic genomic signatures for rapid classification of novel pathogens: COVID-19 case study. PLoS One 15(4).

[35] Quick, J. et al. Multiplex PCR method for MinION and Illumina sequencing of Zika and other virus genomes directly from clinical samples. Nat. Protoc. 12, 1261-1276 (2017).

[36] Rahmatizadeh, S., Valizadeh-Haghi, S., and Dabbagh, A. (2020). The role of artificial intelligence in management of critical COVID19 patients. Journal of Cellular and Molecular Anesthesia, 5(1), 16-22.

[37] World Health Organization. Coronavirus disease 2019 (COVID-19).Situation report 51. https://www.who.int/docs/defaultsource/coronaviruse/situation-reports/20200311sitrep-51-covid19.pdf?sfvrsn=1 ba62e57 10 (2020).

[38] Smith, D. (2020) Trump unveils 'warp-speed' effort to create coronavirus vaccine by year's end", [online], Guardian, May 16, www.theguardian.com/usnews/2020/may/15/trump-coronavirus-warpspeed-vaccine-white-house. 